\title{
Using Subgridding FDTD Method to Incorporate Air Gap Breakdown in the Analysis of Lightning Induced Voltage
}

\author{
Jinliang $\mathrm{He}^{*}$, Shunchao Wang and Rong Zeng
}

State Key Lab of Power Systems, Dept. of Electrical Engineering, Tsinghua University, Beijing 100084, China

\begin{abstract}
A subgridding formulation of Agrawal coupling equations of transmission line with branch and distributed electromagnetic excitation is proposed. This algorithm enables us to solve the transmission line system using different temporal and spatial step on different branches. The method is used to calculate the lightning induced over-voltage on the transmission line installed with air gap component branch. The air gap is modeled by disruptive effect method with small time step, while the transmission line is simulated using large time step. With the help of this method, the simulation of transmission line with air gap component can avoid long time computation. As an example, the protection effectiveness of surge arrester with series air gap is studied using the proposed method.
\end{abstract}

Keywords: Transmission lines, Finite difference time domain methods, Subgridding, Agrawal coupling equations, Air gaps.

\section{INTRODUCTION}

Overvoltage induced by the electromagnetic field of indirect lightning strike is an important reason for power supply failure and destruction of electric devices in low voltage distribution network. Many literatures have studied the mechanism of this electromagnetic phenomenon and its simulation method [1]. When the induced voltage is high enough, it will lead to the air gap discharge of insulators and surge arresters with air gaps. It is necessary to accurately model such air discharge behaviors in order to examine the effectiveness of lightning protection system and the flashover rate of transmission line. Conventional way of modeling the air gap as an ideal switch without considering the time-lag characteristic of breakdown process might result in the inaccuracy of simulation. For better result, the volttime characteristic should be taken into account.

Since air gaps in $10-\mathrm{kV}$ distribution network are very short, the breakdown time is less than $0.1 \mu$ s under high and fast lightning induced overvoltage [2], an appropriate time step of air gap simulation should be very small in order to model the breakdown process. In the inducing mechanism simulation, the time step of electromagnetic field is the same as that of transmission line simulation. The calculation of electromagnetic field generated by lightning is very computational demanding, a small time step means a long computation time.

The most frequently used method of induced overvoltage simulation is Agrawal coupling model [3], which is based on the transmission line assumption of the overhead line. It is usually discretized by FDTD method for numerical solution [4]. In order to maintain a large time step on the transmission line, while using a small time step on the simulation of air gap breakdown, the sub-gridding FDTD

\footnotetext{
*Address correspondence to this author at the State Key Lab of Power Systems, Dept. of Electrical Engineering, Tsinghua University, Beijing 100084, China; Tel: +86-10-62775585; Fax: +86-10-62784709;
} E-mail: hejl@tsinghua.edu.cn technique [5] of transmission line is developed in this paper. At the vicinities of node where air gap component is connected, the line is partitioned into a fine grid, while other part of the line is partitioned into a coarse grid. Based on the wave-equation representation of transmission line, an interface method between coarse and fine grids is proposed in this paper. As an example, the protection effectiveness of surge arrester with series gap is studied by the proposed method.

\section{NUMERICAL MODEL}

\subsection{Analysis of Transmission Line with Air Gap Branch}

With the presence of branch on a transmission line, the Agrawal equations under the condition of perfectly conducting ground are [3]

$$
\begin{aligned}
& \frac{\partial V(x, t)}{\partial x}+R I(x, t)+L \frac{\partial I(x, t)}{\partial t}=E_{L}(x, t) \\
& \frac{\partial I(x, t)}{\partial x}+C \frac{\partial V(x, t)}{\partial t}=I_{\text {avr. } B}
\end{aligned}
$$

where $I(x, t)$ is the current along the line, and $V(x, t)$ is the scattered voltage of the line. The total line voltage $V_{\text {total }}$ is given by

$$
V_{\text {total }}(x, t)=V(x, t)-\int_{0}^{h} E_{T}(x, t) d z
$$

$E_{T}$ and $E_{L}$ are the components of the incident electric field that are transverse to line and parallel to the line, respectively. $I_{\text {avr. } B}$ is the spatial averaged current injected into the line. Denoting the branch current flowing out of the line as $I_{B}$ gives

$$
I_{\text {avr } B}=-\frac{I_{B}}{\Delta x}
$$

where $\Delta x$ is the spatial step used in the discretization.

Suppose the branch is a lumped one, as shown in Fig. (1) and the $V-I$ relation of the branch is known, the recursive 
formula can be obtained easily by discretizing (2) using second order central difference

$$
V_{k}^{n+1}=V_{k}^{n}-\frac{\Delta t}{\Delta x} C^{-1}\left(I_{k}^{n+\frac{1}{2}}-I_{k-1}^{n+\frac{1}{2}}+I_{B}^{n+\frac{1}{2}}\right)
$$

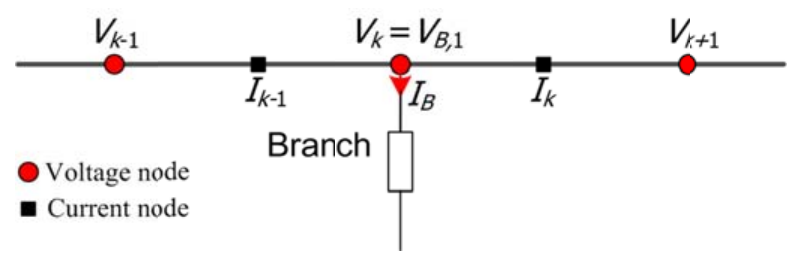

Fig. (1). Illustration of transmission line with a lumped branch.

Given the $V-I$ relation of the branch, $V^{n+1}$ can be obtained from (5).

One of the most commonly used methods in the simulation of time-lag characteristics of short air gap breakdown is the disruptive effect method, which models the breakdown as a cumulative process. In this paper, this method is adopted and the volt-time characteristic of a gap is determined from the following integration equation [2]

$$
D E=\int_{t_{0}}^{t_{b}}\left[V(t)-K_{1}\right]^{K_{2}} d t
$$

where $D E, K_{1}$ and $K_{2}$ are constants for a particular air gap, $t_{0}$ is the time when $V\left(t_{0}\right)=K_{1}$, and $K_{1}$ can be determined by experiments. $K_{2}$ is always assumed to be $1 \sim 2$ and $t_{b}$ is the time when breakdown happens.

In the simulation, (6) is evaluated numerically using the trapezoid integration scheme. Before the breakdown happens, the pre-discharge current is neglected and the air gap is assumed to be a 1-M $\Omega$ resistance. Once the breakdown happens, the air gap is turned into a perfect conductor with zero resistance. The $5-\mathrm{cm}$ rod-rod breakdown data in [2] is employed to fit the parameters in (6). The simulation of a 5-cm air breakdown using (6) with different time steps are presented in Fig. (2). The breakdown happens before the voltage reaches its peak value. The time step has a notable influence on the breakdown time and the peak value of applied voltage.

In the simulation, symmetrical oscillation phenomenon is observed when the switching occurs. Inspired by [6], (5) is modified to the following formula at the time step of switching

$V_{k}^{n+1}=V_{k}^{n}-\frac{\Delta t}{\Delta x} C^{-1}\left(I_{k}^{n+\frac{1}{2}}-I_{k-1}^{n+\frac{1}{2}}+I_{B}^{n+1}\right)$

Using the formulas presented above, the numerical simulation of transmission line with air gap branch can be implemented. It can be seen from Fig. (2) that a small time step is needed to produce accurate result. However, a small time step will bring a high computation cost in the lightning electromagnetic field calculation. In the next section, a subgridding method is proposed to solve this problem.

\subsection{Subgridding Technique}

As discussed in the previous section, the necessity of fine grid arises from the need for accurate simulation of the fast transient of the component connected to transmission line.
The branch, either a lumped component or a transmission line, might need temporal or spatial step smaller than those used on the main line. The uniform small step on all parts means higher computational cost. In this section, we propose a subgridding method, which enables us to use small time step at the part of the transmission line system requiring a fine grid, and large time step at other parts.

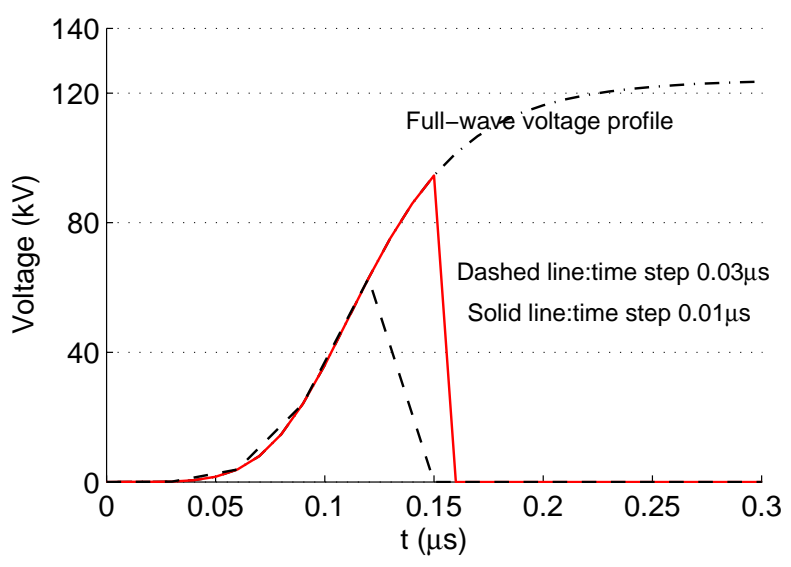

Fig. (2). Profile of the applied voltage on a 5-cm rod-rod air gap. The wavefront time is $0.12 \mu \mathrm{s}$.

The transmission line is partitioned in such a manner that the branch is connected to one of the voltage nodes. The sections adjacent to the branch are partitioned into a fine grid. The geometry of subgridding method is illustrated in Fig. (3a).

Taking partial derivative of equation (1) with respect to $x$ and partial derivative of (2) with respect to $t$ and combining the two equations to eliminate the current term gives

$\frac{\partial^{2} V}{\partial x^{2}}-R C \frac{\partial V}{\partial t}-L C \frac{\partial^{2} V}{\partial t^{2}}=\frac{\partial}{\partial x} E_{L}+\left(L \frac{\partial}{\partial t}+R\right) \frac{I_{B}}{\Delta x}$

Discretizing (8) by the second order central difference method at the $k$ th voltage node gives

$$
\begin{aligned}
& V_{k}^{n+\alpha}=\left[\frac{R C}{2 \alpha \Delta t}+\frac{L C}{(\alpha \Delta t)^{2}}\right]^{-1} \\
& \left(\begin{array}{c}
-L C \frac{-2 V_{k}^{n}+V_{k}^{n-\alpha}}{(\alpha \Delta t)^{2}}+R C \frac{V_{k}^{n-\alpha}}{2 \alpha \Delta t}-\left.\frac{\partial E_{L}}{\partial x}\right|_{k,(n, \alpha)} \\
+\frac{V_{k+\alpha}^{n}-2 V_{k}^{n}+V_{k-\alpha}^{n}}{(\alpha \Delta x)^{2}}-\frac{\left.\left(L \frac{\partial}{\partial t}+R\right) I_{B}\right|_{n}}{2 \alpha \Delta x}
\end{array}\right)
\end{aligned}
$$

where $\alpha=1 / N_{f g}, \ldots, N_{f g} / N_{f g}$, and $N_{f g}$ is the subgridding ratio. $I_{B}$ is the branch current flowing out of the node. $V_{k+\alpha}^{n}$ and $V_{k-\alpha}^{n}$ can be obtained through spatial interpolation

$$
\begin{aligned}
& V_{k-\alpha}^{n}=V_{k}^{n}-\alpha\left(V_{k}^{n}-V_{k-1}^{n}\right) \\
& V_{k+\alpha}^{n}=V_{k}^{n}+\alpha\left(V_{k+1}^{n}-V_{k}^{n}\right)
\end{aligned}
$$

When the branch is a lumped component, the $V-I$ relation of the branch can be represented as

$$
I_{B}=f_{B}\left(V_{k}-\int_{0}^{h} E_{T, k} d z\right),
$$


then, (11) and (9) can be combined together to obtain $V_{k}^{n+\alpha}$. However, when the branch is a transmission line instead of a lumped component, the procedure is not straightforward. In the following section, a method of handling this scenario is proposed.

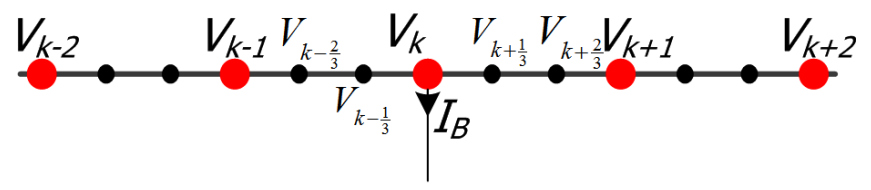

(a) Transmission line Branch

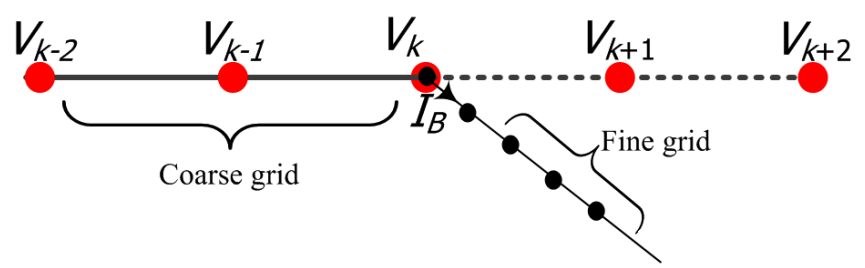

(b) Interface between coarse and fine grid

Fig. (3). The spatial partition of transmission line with both fine and coarse grids, $N_{f g}=3$. Note that the branch line is also horizontal.

\subsection{Subgridding Technique in FDTD Solution of Transmission Line with Different Grids}

Another problem of FDTD simulation of transmission line lies on its high computation cost in handling the frequency-dependent parameters, the convolution should be carried out on every single discretized segment. The more the spatial steps are, the higher the simulation cost is. In spite of the acceleration brought by recursive convolution method, FDTD method is still not suitable for long line simulation. This problem can also be solved by the subgridding method. As illustrated in Fig. (3b), the important part of transmission line is simulated with a fine grid, while the other part is simulated with a coarse grid. This will reduce the number of spatial segments, and correspondingly, the computation time. For the convenience of the deduction, the frequencydependent parameters are neglected in the interface section.

In order to illustrate the deduction, let's consider a more general case: a transmission line is connected with a transmission line branch, as shown in Fig. (3b). We name the horizontal line as the main line, and the vertical line as the branch line. If the section of main line on the right side of the branch is cut down, the system becomes a single transmission line. Although (5) is still correct for this condition, it cannot be evaluated directly, because the relation between $V_{k}$ and $I_{B}$ at the each fine time step is unknown. The FDTD representation of (2) at the terminal of the branch is

$\frac{1}{\Delta x / 2}\left[I_{B, 1}^{n+\frac{1}{2}}-\frac{I_{B}^{n+1}+I_{B}^{n}}{2}\right]+C_{B} \frac{V_{B, 1}^{n+1}-V_{B, 1}^{n}}{\Delta t}=0$

Combining (12) and (5) gives

$V_{B, 1}^{n+1}=V_{k}^{n+1}=V_{k}^{n}+$

$2 \frac{\Delta t}{\Delta x}\left[2 C_{1}+C_{B}\right]^{-1}\left\{\left(I_{k-1}^{n+\frac{1}{2}}-I_{k}^{n+\frac{1}{2}}\right)-I_{B, 1}^{n+\frac{1}{2}}\right\}$

With the help of (13), the voltage recursion on the main line and the branch line can proceed forward.
A subgriding formulation similar to (9) can be obtained

$V_{k}^{n+\alpha}=\left[\frac{R_{B} C_{B}}{2 \alpha \Delta t}+\frac{L_{B} C_{B}}{(\alpha \Delta t)^{2}}\right]^{-1}$

$\left(\begin{array}{l}-L_{B} C_{B} \frac{-2 V_{k}^{n}+V_{k}^{n-\alpha}}{(\alpha \Delta t)^{2}}+R_{B} C_{B} \frac{V_{k}^{n-\alpha}}{2 \alpha \Delta t} \\ +\frac{V_{B, 1+\alpha}^{n}-V_{B, 1}^{n}}{(\alpha \Delta x)^{2}}-\left.\frac{\partial E_{L}}{\partial x}\right|_{k,(n, \alpha)}+\frac{\left.\left(L_{B} \frac{\partial}{\partial t}+R_{B}\right) I_{B}\right|_{n}}{2 \alpha \Delta x}\end{array}\right)$

Combining (9) and (14), the term which contains the branch current $\mathrm{I}_{\mathrm{B}}$ is cancelled out, and $V_{k}^{n+\alpha}$ can be obtained.

In case of multi-conductor transmission line, the formulas developed above can also be used by simply replacing the capacitance and inductance with capacitance and inductance matrices, respectively.

\section{SIMULATION RESULTS}

In practice, the installation of surge arrester with series gap is a promising method to prevent the flashover caused by lightning induced over-voltage [7]. The air gap is aimed to prevent the aging of surge arrester caused by the power frequency voltage. In this paper, a single-conductor overhead line excited by a lightning stroke is studied. One surge arrester with $5-\mathrm{cm}$ series gap is installed to examine the protection effectiveness of the arrester against fast front over-voltage. The grounding resistance of the pole grounding device is assumed to be $30 \Omega$. The geometry of the problem is given in Fig. (4). But we should point out that there is usually a grounding wire of about $10-\mathrm{m}$ long between the arrester and the grounding device, which is not considered in this simulation. Since the proposed approach in this paper is capable of modeling both lumped and distributed components, we will take this factor into account in our future work.

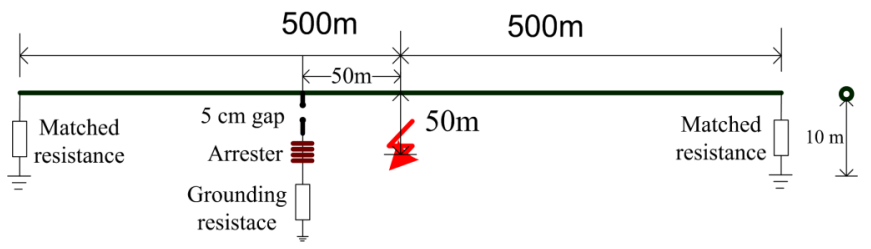

Fig. (4). The example studied in this paper.

Since the $10 \mathrm{kV}$ surge arrester is typically very short, it can be modeled as a lumped nonlinear resistance. NewtonRaphson's method is employed to handle the nonlinearity of the surge arrester. Parameters for the $5 \mathrm{~cm}$ gap are: $D E=6.45$, $K_{1}=40.30$ and $K_{2}=2.0$. Heidler's formula is used to represent the lightning current formula and a fast front lightning current is adopted in this $\operatorname{paper}\left(\tau_{1}=0.1 \mu \mathrm{s}, \tau_{2}=25 \mu \mathrm{s}\right.$ and $I_{p}=42 \mathrm{kA}$ ).

The over-voltage profiles at the point where the surge arrester is installed are observed. The residual voltage on surge arresters with and without a series air gap is compared in Fig. (5). Due to the time-lag characteristic of the breakdown process, there exists a voltage spike before the discharge of surge arrester. This spike might undermine the protection effectiveness of surge arrester. 


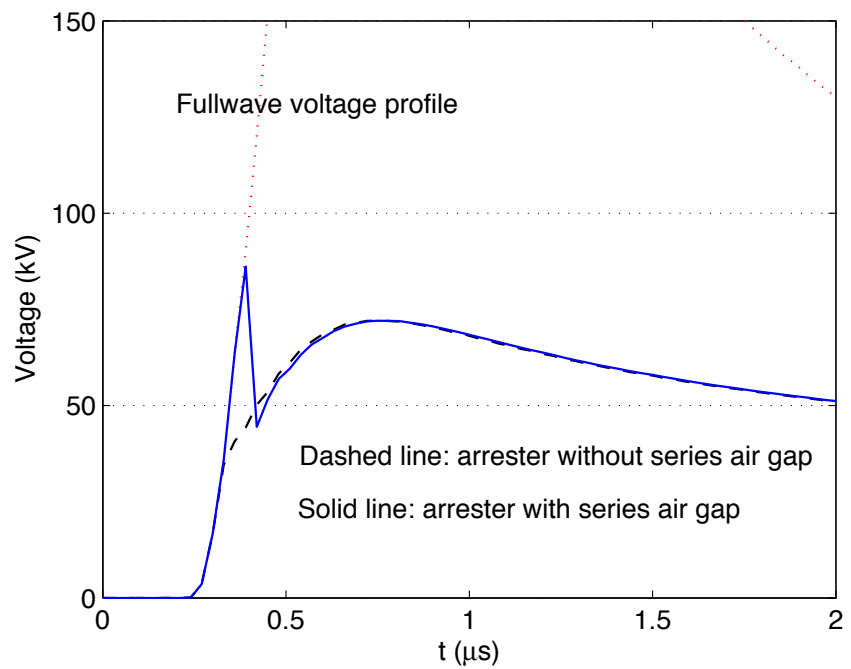

Fig. (5). Profiles of the residual voltage on the arresters with and without air gap, time step is $0.03 \mu \mathrm{s}$.

According to the author's experiences, the largest stable subgridding ratio is $3: 1\left(N_{f g}=3\right)$ for the method proposed in this paper. We still do not understand the cause of instability when the ratio is large. It is probably because of the error introduced at the coarse-fine boundary as noted in [5]. In the simulation, the coarse time step is adopted as $0.03 \mu$ s and the fine time step is $0.01 \mu \mathrm{s}$. The corresponding spatial steps are $10 \mathrm{~m}$ and $10 / 3 \mathrm{~m}$, respectively. Under this lightning surge, the breakdown process lasts about $0.1 \mu \mathrm{s}$ (as shown in Fig. 5). It takes approximately 3 time steps for the coarse grid and 10 time steps for the fine grid. At the vicinities of the voltage node where the surge arrester is installed, the transmission line is partitioned into a fine grid. Inside the fine grid section, (9) is used to evaluate the voltage value at the two adjacent voltage nodes. Equation (7) is used to take the branch into account in the recursive solution.

The simulation results are presented in Fig. (6). While the subgridding method has a good agreement with the fine grid method, the result given by the uniform coarse grid presents a notable difference when compared with them. As can be seen, for the highest voltage value the difference is about $20 \%$.

\section{CONCLUSION}

A general subgridding FDTD formulation for Agrawal coupling equations of transmission line with branch and distributed excitation is proposed in this paper. The two sections adjacent to the branch node are partitioned into a fine grid, while the other part of the transmission line is partitioned into a coarse grid. The proposed method can deal with any type of branch, either a lumped component or a transmission line. It is stable for subgrididing ratio of 3:1 or smaller. The influence of time-lag characteristic of the breakdown process on the lightning protection effectiveness of surge arrester with series air gap is studied. The results obtained using different time steps show notable differences.

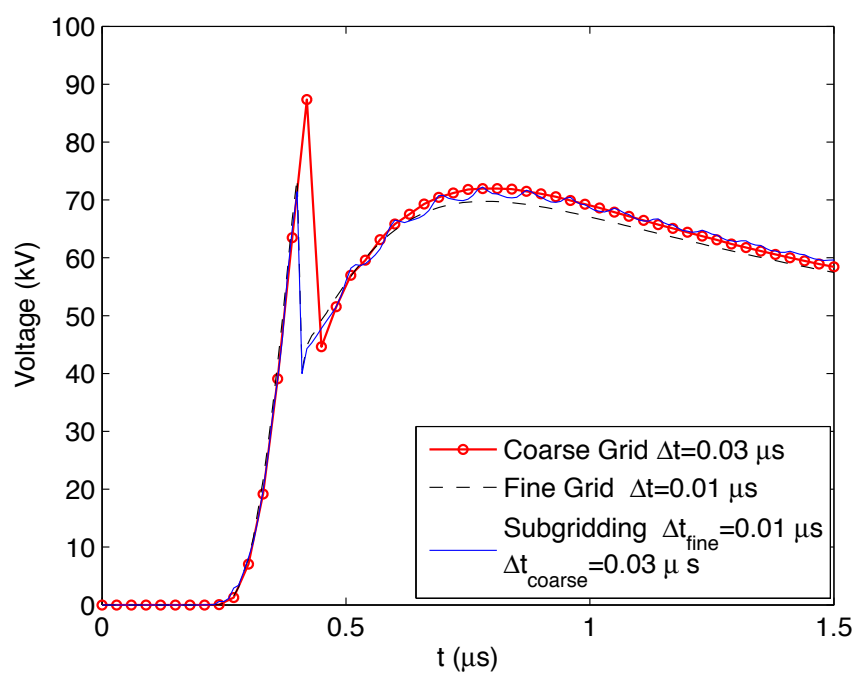

Fig. (6). Comparison of the residual voltage profiles, three calculation modes are considered: coarse grid mode, fine grid mode and subgridding mode.

\section{ACKNOWLEDGEMENT}

This work was supported in part by the National Natural Science Foundation of China under Grant 5073001 and in part by the National Basic Research Program of China (973 Program) under Grant 2009CB724504.

\section{CONFLICT OF INTEREST}

Declared none.

\section{REFERENCES}

[1] Rachidi F, Nucci CA, Ianoz M, Mazzetti C. Influence of a lossy ground on lightning-induced voltages on overhead lines. IEEE Trans Electromag Compat 1996; 38(3): 250-64.

[2] Chowdhuri P, Mishra AK, McConnell BW. Volt-time characteristics of short air gaps under nonstandard lightning voltage waves. IEEE Trans on Power Deliv 1997; 12(1): 470-6.

[3] Agrawal AK, Price HJ, Gurbaxani SH. Transient response of multiconductor transmission lines excited by a nonuniform electromagnetic field. IEEE Trans Electromag Compat 1980; 22: 119-29.

[4] Orlandi A, Paul CR. FDTD analysis of lossy, multiconductor transmission line terminated in arbitrary load. IEEE Trans 1996; 38(3): 388-98.

[5] Zivanovic SS, Yee KS, Mei KK. A subgridding method of timedomain finite-difference method to solve Maxwell's equations. IEEE Trans Microw Theory 1991; 39 (3): 471-9.

[6] Marti JR, LinJ. Suppression of numerical oscillations in the EMTP power systems. IEEE Trans Power Syst 1989; 4(2) 739-47.

[7] Gu S, Chen W, He J, Shen H, Zhang S. Monitoring results of the effectiveness of surge arrester spacingson distribution line protection. IEEE Trans Power Deliv 2007; 22 (4): 2191-8. 